\title{
marges Marges
}

revue d'art contemporain Revue d'art contemporain

27 | 2018

Ce que fait le concept à l'œuvre

\section{Kenneth Goldsmith, L'Écriture sans écriture du langage à l'âge numérique}

Trad. fr. F. Bon, Paris, Jean Boîte Éditions, 2018, 248 p.

Émeline Jaret

\section{OpenEdition}

\section{Journals}

Édition électronique

URL : https://journals.openedition.org/marges/1707

DOI : $10.4000 /$ marges. 1707

ISSN : 2416-8742

\section{Éditeur}

Presses universitaires de Vincennes

Édition imprimée

Date de publication : 25 octobre 2018

Pagination : 208-209

ISBN : 9782842928339

ISSN : 1767-7114

\section{Référence électronique}

Émeline Jaret, « Kenneth Goldsmith, L'Écriture sans écriture du langage à l'âge numérique », Marges

[En ligne], 27 | 2018, mis en ligne le 25 octobre 2018, consulté le 06 janvier 2022. URL : http:// journals.openedition.org/marges/1707 ; DOI : https://doi.org/10.4000/marges.1707 


\section{Kenneth Goldsmith, \\ L'Écriture sans écriture - \\ du langage à l'âge numérique}

Trad. fr. F. Bon, Paris, Jean Boîte Éditions, 2018, 248 p.

Publié en 2011, l'ouvrage de Kenneth Goldsmith est rapidement devenu un classique de la littérature conceptuelle américaine et sa traduction française par François Bon vient de paraître. Poète, artiste et théoricien, Goldsmith centre son essai sur la notion d'Uncreative Writing - ici traduite comme une " écriture sans écriture » - qu'il définit comme une conséquence de la révolution numérique. Comparant le bouleversement provoqué dans notre rapport à l'écriture par le passage à l'ère numérique au $21^{\mathrm{e}}$ siècle à la révolution engendrée dans la peinture par l'invention de la photographie au $19^{\mathrm{e}}$ siècle, cet essai s'interroge sur « le langage à l'âge numérique », afin de repenser la créativité dans un contexte où le développement d'Internet $\mathrm{a}$ induit de profondes modifications du langage.

Dans les premières lignes de l'introduction Goldsmith postule que « Le monde est rempli de textes, plus ou moins intéressants; je n'ai aucune envie de lui en ajouter un de plus.» (p. 9). La paraphrase assumée de Douglas Huebler - qui affirme dès 1969 que « le monde est rempli d'objets [...] »- sert à justifier ce que Goldsmith qualifie de nouvelle condition de l'écriture; à savoir l'idée qu'il n'est désormais plus nécessaire de créer de nouveaux textes ex nihilo mais qu'il s'agit bien d'“ apprendre à négocier » la quantité de textes existants et rendus disponibles par Internet. Goldsmith s'appuie ici sur le concept de "génie non original» (Unoriginal genius) forgé par Marjorie Perloff pour requalifier l'auteur de « récupérateur de langage » (p. 12) et transformer l'écrivain en programmeur. Selon lui, le renouvellement de l'écriture doit se faire par l'appropriation de textes existants, littéraires ou non, choisis, réarrangés et plagiés, dans une sorte de généralisation ou d'extension extrême du geste devenu naturel de copiercoller. C'est donc la notion d'auteur qui est renouvelée pour faire face aux nouvelles exigences du langage à l'âge numérique, Goldsmith proposant d'aborder l'auctorialité de façon conceptuelle: «les meilleurs auteurs du futur seront peut-être ceux qui auront écrit les meilleurs programmes pour manipuler, analyser et distribuer des protocoles à base de langage » (p. 18). L'Uncreative Writing remet ainsi en cause l'originalité comme condition nécessaire de l'œuvre et la déclare obsolète. Sur ce point et comme beaucoup d'autres avant lui, Goldsmith dénonce un retard de la littérature par rapport aux autres arts, tant visuels que musicaux, qui se sont depuis longtemps libérés des notions de génie et d'originalité. L'Uncreative Writing 
est ainsi proposée comme une voie pour sortir de l'« impasse de l'écriture » (p. 15). L'ouvrage rassemble treize essais afin de «de cartographier ces territoires, d'en définir les terminologies, et de créer des contextes [...] dans lesquels ces œuvres peuvent être situées et discutées" (p. 18). Les cinq premiers chapitres se concentrent sur les sources, les modèles et les contextes d'expansion de l'Uncreative Writing, tentant de répondre à: Comment? Où? Pourquoi? Goldsmith y analyse l'essor d'Internet tout en dressant une généalogie de l'Uncreative Writing, l'inscrivant à la suite d'expérimentations artistiques et littéraires, telles que le situationnisme, la poésie concrète et l'art conceptuel, mais aussi dans celle de l'ouvrage de Walter Benjamin, Paris, capitale du $X I X^{e}$ siècle, qu'il qualifie de " feuille de route littéraire pour l'appropriation» (p.120). Dans le chapitre six, Goldsmith s'appuie d'ailleurs sur les exemples d'Andy Warhol et de Sol LeWitt pour rappeler que les arts visuels «ont depuis longtemps considéré la non-créativité comme une des pratiques créatives » (p. 129). Les chapitres 7 à 10 proposent, quant à eux, des modalités de mise en œuvre de l'Uncreative Writing, analysant le plagiat intentionnel et revenant sur le rôle de l'archive et des réseaux sociaux dans le renouvellement de l'écriture. Le onzième chapitre confirme la dimension pédagogique de cet ouvrage en se concentrant sur la manière d'enseigner la création littéraire - à partir d'un séminaire que Goldsmith a lui-même donné à l'Université de Pennsylvanie. Enfin, les deux derniers essais sont des manifestes pour l'Uncreative Writing, définie comme conséquence d'une dévalorisation du langage à l'âge numérique. Suite à un phénomène d'« industrialisation du langage » (p. 232), l'écrivain est transformé en « producteur, éditeur et diffuseur» (p. 233) et Goldsmith lui demande d'exploiter les possibilités offertes par la technologie dans un monde numérique lui-même devenu « un langage provisoire, passager et déprécié, simple matériau pour être déblayé, reformaté, stocké et repris dans n'importe quelle forme adaptée » (p. 231).

L'Uncreative Writing consiste ainsi à accorder toute son importance non plus au contenu du texte mais à son contexte et à ses modalités de composition, au processus plus qu'à l'écriture même. En ce sens, et Goldsmith le rappelle tout au long de son ouvrage, cette notion est l'héritière d'une longue tradition littéraire et artistique du $20^{\mathrm{e}}$ siècle, qui passe par les collages de dada, les cut-ups de Brion Gysin ou William Burroughs, l'intertextualité de Georges Perec, le situationnisme, les protocoles de l'art conceptuel, les appropriations de la Pictures Generation, etc. L'écriture à l'âge numérique doit désormais être le fruit d'un «transfert littéral de langage d'un lieu à un autre» (p. 11). Cet essai est en effet éclairant sur bien des points et la traduction qu'en propose Bon reflète le défi qu'impose la notion d'Uncreative Writing pour la littérature aujourd'hui. Traduire Uncreative Writing par "écriture sans écriture » est une décision certes compréhensible mais discutable, proposant un équivalent adéquat mais non exact, dans la mesure où il ne recouvre pas l'entière signification du vocable anglais uncreative. Bon, qui est lui-même écrivain et enseignant de création littéraire à l'ENSAPC (Cergy), choisit ici de proposer une traduction littéraire et non littérale - ce que démontre également l'appareil de notes -, injectant un peu de sa créativité dans cet essai-manifeste pour une écriture non-créative.

\section{Émeline Jaret}

